\title{
ÁP DƯNG MÔ HÌNH MIKE SHE KÊTT HỢP SỬ DỤNG SẢN PHẨM MƯA DỬ BÁO IFS DỰ BÁO LƯU LƯợNG ĐẾN HỔ LƯU VỰC SÔNG TRÀ KHÚC-SÔNG Vệ
}

\author{
Trần Hồng Thái ${ }^{12}$, Đoàn Quang Trí' ${ }^{2}$ Trần Đỗ Thủy Tuyên ${ }^{3}$, \\ Ngô Thanh Tâm², Bùi Thị Dịu ${ }^{2}$
}

Tóm tắt: Nghiên cứu đã ứng dụng được sản phẩm mua dụ báo IFS làm đầu vào cho mô hình MIKE SHE phục vu tính toán và dụ báo lư lượng nước đến cho hai hồ chứa trên lưu vưc sông Trà Khúc-Sông Vệ. Quá trình hiệu chỉnh và kiểm định mô hình thủy văn sử dụng số liệu của các trận lũ điển hình trên luu vực tù̀ năm 2013-2016. Kết quả hiệu chỉnh và kiểm định mô hình chỉ ra sự tuong đồng về pha và biên độ dao động giữa lưu luọng tính toán và thực đo tại hai trạm Sonn Giang và An Chi. Kết quả đánh giá kiểm định và dụ báo luu luọng nước đến hai hồ ĐăkĐrinh và hồ Nước Trong cho kết quả rất tốt theo các chỉ tiêu đánh giá $r, R^{2}$, NSE, RMSE, MAE, PBIAS. Kết quả dụ báo thư nghiệm cho hai trận lũ điển hình năm 2017 cho thấy chất lượng dụ báo lưu lượng đến hai hồ đã tăng lên đáng kể. Đây sẽ là một công cu hiệu quả phuc vu tốt cho công tác dụ báo nghiệp vu trong tuoong lai.

Tù khóa: IFS, MIKE SHE, Trà Khúc-Sông Vệ.

Ban Biên tập nhận bài: 09/11/2018 Ngày phản biện xong: 25/12/2018 Ngày đăng bài: 25/01/2019

\section{1. Đặt vấn đề}

Để nâng cao chất lượng mô phỏng dòng chảy từ mưa, xu hướng mới trên thế giới hiện nay là sử dụng các mô hình thủy văn có thông số phân bố. Các ứng dụng theo xu hướng này đang được nghiên cứu phát triển và áp dụng trong dự báo thủy văn. Đầu vào của các mô hình thủy văn có thông số phân bố là các số liệu thông tin địa lý (GIS), viễn thám, ước lượng mưa từ vệ tinh, ra đa hay mưa dự báo số trị. Đại học Tokyo bước đầu phát triển mô hình thủy văn phân bố sử dụng vốn từ Quỹ nước và năng lượng (Water and Energy Budget-based Distributed Hydrological Model, WEB-DHM) mô phỏng cho năm 2006 với dòng chảy sông Hồng trong khuôn khổ Chương trình Chu trình nước châu Á (Asia Water Cycle Initiative, AWCI) [29]. Mô hình này dựa trên số liệu viễn thám và số liệu mưa bề

${ }^{1}$ Tổng cuc Khi tượng Thủy văn, Hà Nội

${ }^{2}$ Tạp chi Khi tuợng Thủy văn, Tổng cuc Khi tuợng Thủy văn, Hà Nội

${ }^{3}$ Truờng Đại học Tài Nguyên và Môi trường Hà Nội

Email: doanquangtrikttv@gmail.com mặt. Trong một vài năm trở lại đây việc ứng dụng mô hình số trị trong nghiên cứu khí tượng nói chung và dự báo thời tiết nói riêng đã được phát triển mạnh mẽ ở nước ta. Hàng loạt các mô hình số trị khu vực đã được nghiên cứu áp dụng nhằm dự báo thời tiết, đặc biệt là dự báo các hiện tượng cực đoan như mưa lớn, bão, áp thấp nhiệt đới, ... Mô hình khu vực hạn chế RAMS (Regional Area Model System) đã được nghiên cứu và đang được thử nghiệm trong dự báo thực tế tại Trường Đại học Khoa học Tự nhiên. Đây là một mô hình có nhiều ưu điểm trong mô phỏng các quá trình lớp biên. Điều kiện ban đầu và điều kiện biên cho mô hình RAMS được lấy từ sản phẩm của mô hình AVN/NCEP (Mỹ). Từ nhiều năm nay, Trung tâm dự báo KTTV Trung ương (TTDBTU') nay là Trung tâm Dự báo Khí tượng Thủy văn quốc gia (TTDBQG) đã đầu tư nghiên 
cứu và đưa vào sử dụng một số công nghệ hiện đại trong dự báo mưa số trị. Trong đó, TTDBQG đã được Bộ Tài nguyên và Môi trường đầu tư dự án mua các sản phẩm (dạng ảnh có sẵn trên trang web) và số liệu dạng số (dạng mã GRIB truyền qua mạng Internet) của Trung tâm Dự báo hạn vừa Châu Âu (ECMWF) để phục vụ công tác dự báo nghiệp vụ khí tượng hạn ngắn - hạn dài (Hình 1). Đây là nguồn số liệu dự báo khí tượng toàn cầu toàn diện nhất mà TTDBQG có thể khai thác. Trong nghiên cứu này đã sử dụng sản phẩm mưa dự báo IFS làm đầu vào cho việc mô phỏng dòng chảy tới hồ trên lưu vực sông Trà KhúcSông Vệ. Từ khi các hệ thống hồ chứa ở Việt Nam đi vào hoạt động, dự báo lũ đã trở thành một nhiệm vụ quan trọng phục vụ việc điều hành hồ chống lũ cho hạ du và sản xuất điện năng. Đây là một vấn đề hết sức phức tạp được nhiều nhà nghiên cứu trong nước quan tâm.

Hiện nay rất nhiều mô hình toán thủy văn, thủy lực đã được nghiên cứu để ứng dụng trên các hệ thống sông miền Trung, nhưng chỉ rất ít trong số đó có thể ứng dụng được trong nghiệp vụ dự báo hàng ngày: mô hình Wetspa kết hợp với mô hình thủy lực HEC-RAS đã nghiên cứu và ứng dụng thành công trên lưu vực sông $\mathrm{Vu}$
Gia - Thu Bồn [8]; bộ mô hình MIKE (MIKE NAM, MIKE 11-GIS, MIKE FLOOD) cho sông Thạch Hãn [26]; mô hình thủy lực TELEMAC2D kết hợp với MIKE NAM cho vùng hạ lưu hệ thống sông Trà Khúc - Sông Vệ [28]. MIKE SHE được sử dụng nhiều nơi trên thế giới, tuy nhiên ở Việt Nam những nghiên cứu ứng dụng MIKE SHE còn chưa nhiều [13, 22]. Các nghiên cứu ở lưu vực sông Trà Khúc - Sông Vệ chủ yếu sử dụng mô hình thông số tập trung MIKE NAM để mô phỏng dòng chảy, chưa có nghiên cứu nào ứng dụng mô hình thông số phân bố MIKE SHE được sử dụng trong nghiên cứu dự báo nghiệp vụ. Mô hình MIKE SHE có khả năng mô phỏng quá trình mưa-dòng chảy trên từng ô lưới theo không gian, phù hợp với việc sử dụng số liệu mưa số trị.

Mục đích của nghiên cứu này: (1) Úng dụng sản phẩm của mô hình IFS làm đầu vào cho mô hình thủy văn; (2) Nghiên cứu ứng dụng mô hình thủy văn thông số phân bố MIKE SHE tính toán, dự báo lưu lượng nước đến hai hồ ĐăkĐrinh và hồ Nước Trong; (3) Đánh giá khả năng áp dụng của mô hình trong bài toán phục vụ công tác nghiệp vụ dự báo.

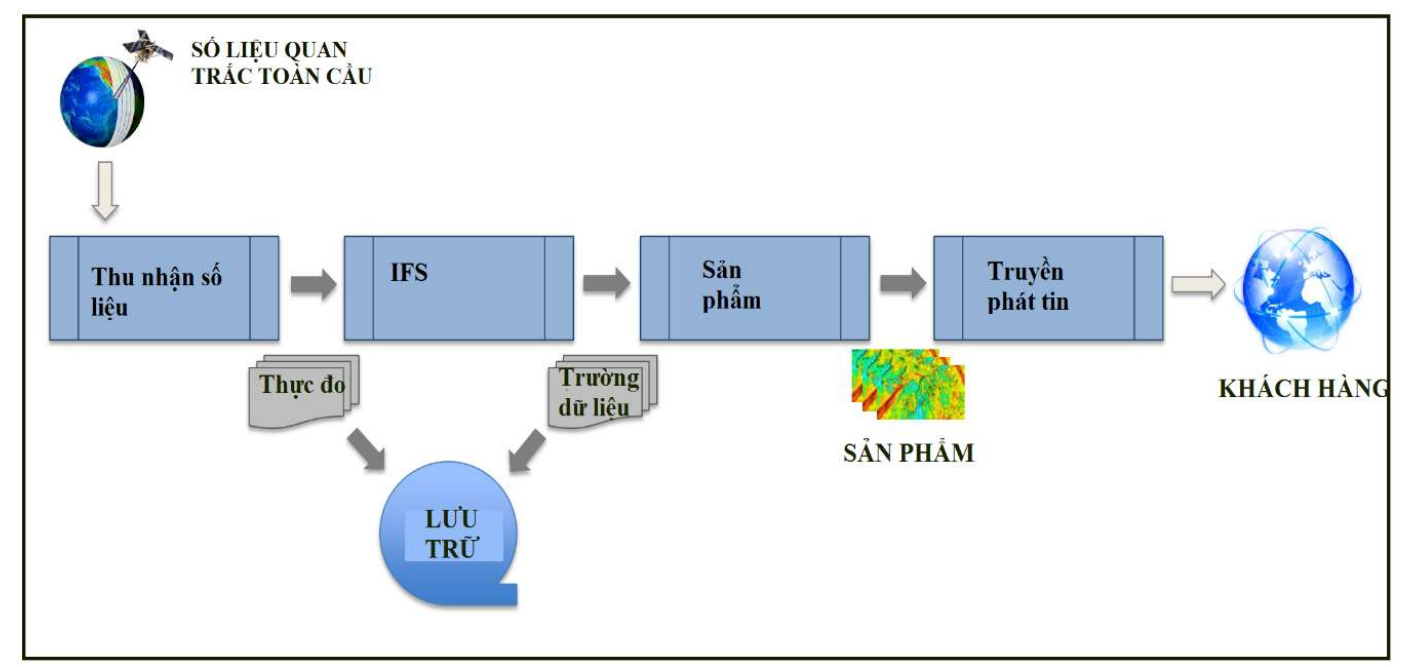

Hình 1. So đồ quá trình thu - nhận dũ liệu của IFS

\section{Phương pháp nghiên cứu và thu thập tài liệu}

2.1 Giới thiệu vị trí khu vục nghiên cứu
Lưu vực sông Trà Khúc -Sông Vệ tỉnh Quảng Ngãi thuộc khu vực Trung Trung Bộ (hình 2). Ranh giới lưu vực sông Trà Khúc: phía Bắc giáp 
lưu vực sông Trà Bồng, phía Nam giáp lưu vực sông Vệ, phía Tây giáp lưu vực sông Sê San, phía Đông giáp biển Đông. Lưu vực sông nằm trên địa bàn các huyện Sơn Hà, Sơn Tây, Trà Bồng, Ba Tơ, Sơn Tịnh, Tư Nghĩa, Nghĩa Hành, thị xã Quảng Ngãi và một phần huyện Kon Plong thuộc tỉnh Kon Tum.Với tổng diện tích $4.600 \mathrm{~km}^{2}$ sông Trà Khúc - sông Vệ có mô đuyn dòng chảy trung bình nhiều năm đạt $70 \div 80$ $1 / \mathrm{s} / \mathrm{km}^{2}$. Trên sông Trà Khúc tại Sơn Giang đạt $193 \mathrm{~m}^{3} / \mathrm{s}$ tương ứng với mô số dòng chảy là 71,3 $1 / \mathrm{s} / \mathrm{km}^{2}$ và tổng lượng dòng chảy 6,1 tỷ $\mathrm{m}^{3}$ nước. Mưa lũ lớn ở vùng ven biển miền Trung nói chung và hệ thống sông Trà Khúc - Sông Vệ nói riêng thường do các hình thế thời tiết như: bão, áp thấp nhiệt đới, không khí lạnh, dải hội tụ nhiệt đới và các nhiễu động nhiệt đới khác như gió đông (chủ yếu là sóng đông) gây nên. Đặc điểm tự nhiên của sông Trà Khúc chịu sự chi phối của điều kiện địa hình trên lưu vực sông. Phần thượng lưu là các dãy núi có địa hình dốc nên sông ở đoạn này có hệ số dòng chảy lớn, thời gian tập trung nước nhanh. Lũ trên sông Trà Khúc thường xảy ra rất nhanh, biên độ từ 3-5 m; lũ thường lên trong một ngày, ngắn nhất là 12 giờ, dài nhất là 71 giờ; cường suất nước lên thường là $30-40 \mathrm{~cm} /$ giờ, cao nhất là $78 \mathrm{~cm} /$ giờ. Vào mùa khô (từ tháng 1 đến tháng 8 ), lưu lượng trung bình chỉ còn nhỏ hơn 100 m³/s.

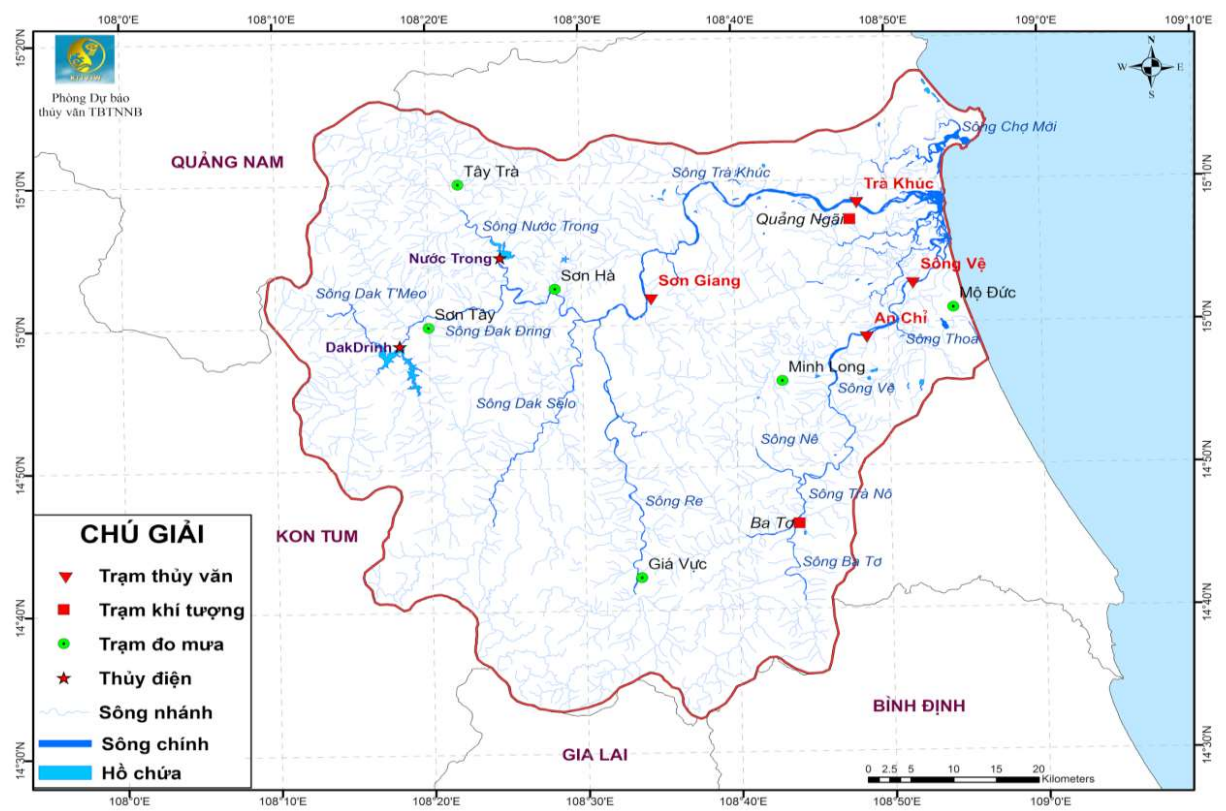

Hình 2. Bản đồ mạng lưới trạm khí tương thủy văn lưu vục Trà Khúc - Sông Vệ

\subsection{Thu thập tài liệu}

Trong nghiên cứu này một số dữ liệu đầu vào được sử dụng như sau:

Số liệu khí tượng: Sử dụng số liệu mưa số trị từ mô hình IFS với thời đoạn 6 giờ phục vụ làm đầu vào cho mô hình thủy văn MIKE SHE; Số liệu mưa thực đo tại các trạm Khí tượng, thủy văn trên lưu vực phục vụ việc hiệu chỉnh và kiểm định mô hình thủy văn từ năm 2012-2016.

Số liệu thủy văn: Sử dụng số liệu của 02 trạm thủy văn phục vụ cho quá trình hiệu chỉnh và kiểm định mô hình bao gồm: Sơn Giang và $\mathrm{An}$ Chỉ với chuỗi số liệu từ năm 2012-2016. Số liệu lưu lượng nước đến hai hồ ĐăkĐrinh và hồ Nước Trong từ năm 2016 tới nay phục vụ kiểm định và đánh giá dự báo lưu lượng nước đến hồ.

Số liệu địa hình: sử dụng bản đồ số độ cao (DEM) cho toàn bộ lưu vực Trà Khúc-Sông Vệ tỉ lệ 1:12500 phục vụ cho việc phân chia lưu vực trong mô hình MIKE SHE [27]. Bản đồ sử dụng đất toàn quốc (Atlas Việt Nam) trong đó tiến hành thiết lập phân loại đất phù hợp đối với lưu 
vực nghiên cứu. Bản đồ thảm phủ thực vật cho khu vực nghiên cứu được xử lý từ bản đồ thảm phủ thực vật toàn quốc.

\subsection{Giới thiệu mô hình MIKE SHE}

MIKE SHE là một mô hình thủy văn thông số phân bố dựa trên các quá trình vật lý được tích hợp đầy đủ, mô hình này có khả năng mô phỏng các quá trình thủy văn tại một điểm, trên một diện rộng, quy mô lưu vực, quá trình vận chuyển các phần tử, và có thể được liên kết với MIKE 11 để mô phỏng các mối quan hệ trong lưu vực sông. Mô hình MIKE SHE ban đầu được phát triển bởi ba tổ chức châu Âu (Viện Thủy lực Đan Mạch, Viện Thủy văn Anh và một công ty tư vấn Pháp SOGREAH) vào năm 1977. DHI đã dẫn đầu trong việc phát triển và nghiên cứu MIKE SHE để cải thiện và bổ sung $[9,10]$. Bản chất vật lý của mô hình bao gồm quá trình mô phỏng địa hình tự nhiên và các đặc điểm của lưu vực như thực vật, đất và thời tiết.
Bản chất phân tán của mô hình cho phép người dùng thay đổi các bộ tham số theo không gian và thời gian như: sử dụng đất, hệ thống thoát nước, dữ liệu thời tiết và bốc hơi, các giá trị dòng chảy trên mặt đất. Sự phân bố không gian được thực hiện thông qua một mạng lưới trực giao cho phép phân loại theo chiều ngang hoặc dọc, được áp dụng trong mỗi bộ tham số $[1,2]$. Phân phối thời gian cho phép người dùng thay đổi các tham số theo thời gian hoặc đặt các giá trị không đổi cho các tham số cho toàn bộ thời gian mô phỏng. Người dùng cũng có thể thay đổi độ phức tạp quá trình mô phỏng bằng cách điều chỉnh thiết lập mô-đun của mô hình trong giao diện người dùng. MIKE SHE bao gồm các môđun: Dòng chảy tràn $(\mathrm{OF})$, sông và hồ $(\mathrm{OC})$, dòng chảy chưa bão hoà (UZ), bốc thoát hơi nước $(\mathrm{ET})$, và dòng chảy bão hoà (SF) (Hình 3). Nếu mô-đun dòng chảy bão hòa được chọn thì trong đó sẽ bao gồm mô đun UZ và ET.

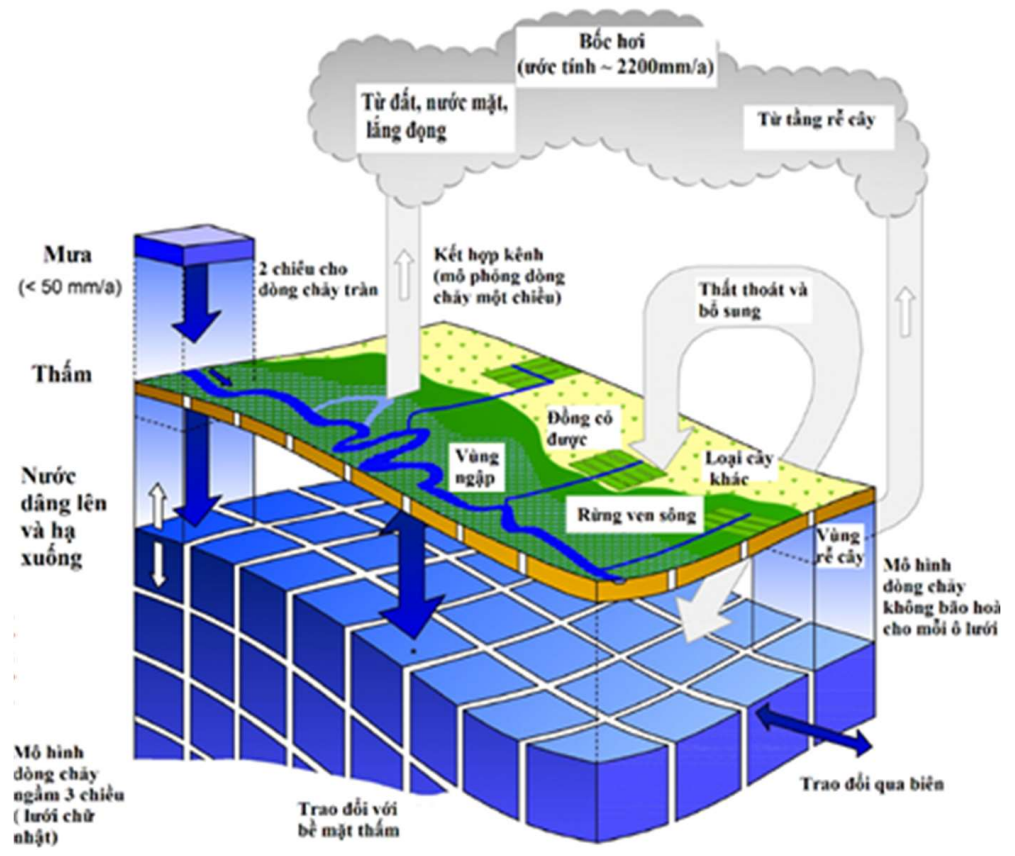

Hình 3. So đồ mô phỏng trong mô hình MIKE SHE[9, 10]

\section{4 Đánh giá mô hình}

Việc ứng dụng các công cụ mô hình hóa trong quản lý tài nguyên nước đang ngày một phổ biến và hiệu quả nhằm mục đích dự đoán những thay đổi trong tương lai về khí hậu, thay đổi về tình hình sử dụng đất và cây trồng cũng như phục vụ quản lý số lượng và chất lượng tài nguyên nước và đất tốt hơn[17]. Tuy nhiên, để đánh giá khả năng của các mô hình này để đưa ra dự đoán một cách chính xác vẫn cần được kiểm nghiệm bằng 
các chỉ số đánh giá mô hình cho phù hợp $[7,11$, $14,17,18,24]$. Trongbài báo này đã sử dụng 05 chỉ số để đánh giá chất lượng của mô hình so với số liệu quan trắc thực tế bao gồm: $r, \mathrm{R}^{2}$, NSE, RMSE và PBIAS.

Hệ số tương quan(Correlation coefcient) (r)

Hệ số tương quan (r) đôi khi bị nhầm lẫn với $\mathrm{R}^{2}$. Cả hai đều được sử dụng trong việc đánh giá các mô hình thủy văn. Tương quan Pearson là thước đo được sử dụng phổ biến nhất của chuỗi liên kết thống kê. Nó cung cấp ước tính số lượng của chuỗi thống kê đồng biến giữa số liệu tính toán và thực đo [3]. Hệ số tương quan (r) được tính theo công thức 1 .

$$
\mathrm{r}=\frac{\sum_{\mathrm{i}=1}^{\mathrm{N}}\left(\mathrm{Q}_{\mathrm{TD}}-\overline{\mathrm{Q}}_{\mathrm{TD}}\right)\left(\mathrm{Q}_{\mathrm{tt}}-\overline{\mathrm{Q}}_{\mathrm{TD}}\right)}{\sqrt{\sum_{\mathrm{i}=1}^{\mathrm{N}}\left(\mathrm{Q}_{\mathrm{TD}}-\overline{\mathrm{Q}}_{\mathrm{TD}}\right)^{2}\left(\mathrm{Q}_{\mathrm{tt}}-\overline{\mathrm{Q}}_{\mathrm{TD}}\right)^{2}}}
$$

Trong đó $\overline{\mathrm{Q}}_{\mathrm{TD}}$ là giá trị lưu lượng thực đo; là giá trị lưu lượng trung bình thực đo; $\mathrm{Q}_{\text {Tт }}$ là giá trị lưu lượng tính toán; $\mathrm{N}$ là số lượng giá trị thực đo.

\section{$\left(R^{2}\right)$}

Hệ số xác định (Coefcient of determination)

Hệ số xác định được định nghĩa là bình phương của hệ số tương quan [15]. Phương trình tính toán giá trị $\mathrm{R} 2$ được trình bày trong công thức 2 . Các giá trị $\mathrm{R}^{2}$ nằm trong khoảng từ 0 đến 1. Các giá trị mô phỏng bằng 1 đại diện cho một phân bố hoàn hảo giữa các giá trị mô phỏng và thực đo, trong khi các giá trị bằng 0 chỉ ra không có sự tương quan. Một sự bất lợi lớn của $\mathrm{R}^{2}$ là, sẽ có sự không rõ ràng trong các kết quả nếu như mô hình đánh giá thiên cao hoặc thiên thấp các kết quả [15]. Mặc dù điều này có thể dễ dàng được phân loại bằng cách so sánh trực quan các kết quả mô phỏng và thực đo [22].

$$
\mathrm{R}^{2}=\frac{\sum_{\mathrm{i}=1}^{\mathrm{N}}\left(\mathrm{Q}_{\mathrm{TD}}-\overline{\mathrm{Q}}_{\mathrm{TD}}\right)\left(\mathrm{Q}_{\mathrm{TT}}-\overline{\mathrm{Q}}_{\mathrm{TD}}\right)}{\sqrt{\sum_{\mathrm{i}=1}^{\mathrm{N}}\left(\mathrm{Q}_{\mathrm{TD}}-\overline{\mathrm{Q}}_{\mathrm{TD}}\right)\left(\mathrm{Q}_{\mathrm{TT}}-\overline{\mathrm{Q}}_{\mathrm{TD}}\right)}}
$$

\section{(NSE)}

Hệ số Nash (Nash-Sutcliffe effciency)

Nash và Sutcliffe (NSE) (1970) [23] là phương pháp phổ biến và đáng tin cậy nhất để đánh giá chất lượng của các mô hình thủy văn. Chỉ số NSE được tính toán theo công thức 3. Các giá trị NSE nằm trong khoảng từ 0 đến 1 . Một sự phù hợp tốt nhất được biểu thị bằng giá trị 1 trong khi 0 biểu thị sự phù hợp kém. Andersen và cs (2001) [4] đã chỉ ra rằng NSE trong khoảng từ 0,5 đến 0,95 thể hiện kết quả mô phỏng tốt. Hiện nay trong các nghiên cứu đánh giá mô hình thủy văn việc sử dụng chỉ tiêu NSE như là một công cụ phổ biến trong hầu hết các nghiên cứu [19].

$$
\mathrm{NSE}=1-\frac{\sum_{\mathrm{i}=1}^{\mathrm{N}}\left(\mathrm{Q}_{\mathrm{iTD}}-\mathrm{Q}_{\mathrm{iTT}}\right)^{2}}{\sum_{\mathrm{i}=1}^{\mathrm{N}}\left(\mathrm{Q}_{\mathrm{iTD}}-\overline{\mathrm{Q}}_{\mathrm{iTD}}\right)^{2}}
$$

\section{Sai số bình phương trung bình (Root mean square error) (RMSE)}

Để có một kết quả đánh giá hiệu quả việc lựa chọn cẩn thận các biến cho RMSE đã được đưa ra bởi Moriasi và cs (2007) [18]. Đây là điều kiện tiên quyết được tính toán bởi phương trình 4, đánh giá mức độ phù hợp giữa số liệu thực đo và tính toán. Các giá trị thường được sử dụng là lưu lượng đỉnh lũ, thời gian đạt đỉnh và tổng lượng. Các thông số khác cũng có thể được sử dụng tùy thuộc vào mô hình và mục tiêu đặt ra.

$$
\operatorname{RMSE}=\left(\frac{\sum_{\mathrm{i}=1}^{\mathrm{N}}\left(\mathrm{Q}_{\mathrm{iTD}}-\mathrm{Q}_{\mathrm{iTT}}\right)^{2}}{\mathrm{~N}}\right)^{\frac{1}{2}}
$$

\section{Sai số trung bình tuyệt đối (Mean absolute error) (MAE)}

MAE được tính toán bởi phương trình 5 , được sử dụng để đánh giá sự khác biệt giữa dữ liệu thực đo và tính toán từ mô hình. Giá trị MAE bằng 0 chỉ ra sự phù hợp tốt nhất. Đã có rất nhiều nghiên cứu áp dụng MAE để đánh giá chất lượng các mô hình thủy văn như HEC-HMS, DENFIS, ARX, HEC-1, LSM, SWAT [5, 6, 16, 20, 28].

$$
\mathrm{MAE}=\frac{\sum_{\mathrm{i}=1}^{\mathrm{N}}\left|\mathrm{Q}_{\mathrm{iTD}}-\mathrm{Q}_{\mathrm{iTT}}\right|}{\mathrm{N}}
$$

\section{Phần trăm BIAS (PBIAS)}

Gupta và cs (1999) [12] đã chỉ ra rằng PBIAS 
là một loại phân tích sai số thống kê định lượng khả năng mô phỏng các giá trị của mô hình để đánh giá giới hạn dao động của chuỗi dữ liệu quan trắc được. PBIAS có thể được tính toán bằng cách sử dụng công thức 6 .

$$
\text { PBIAS }=\frac{\sum_{\mathrm{i}=1}^{\mathrm{N}}\left(\mathrm{Q}_{\mathrm{iTD}}-\mathrm{Q}_{\mathrm{iTT}}\right) \times 100}{\sum_{\mathrm{i}=1}^{\mathrm{N}} \mathrm{Q}_{\mathrm{iTD}}}
$$

\subsection{Thiết lập các thông số đầu vào cho mô hình MIKE SHE}

Địa hình khu vực tính toán được xử lý từ số liệu DEM khu vực Trà Khúc-Sông Vệ bởi công cụ hỗ trợ của ArcGIS. Mô hình MIKE SHE cho phép tiếp nhận dữ liệu dạng lưới Dfs2, dạng đường/điểm shp file (Hình $4 \mathrm{a}$ ). Miền tính của lưu vực nghiên cứu được xác định danh giới cho cả vùng (Hình 4b). Số liệu mưa, bốc hơi được lấy từ mô hình số trị IFS, cách xử lý và tích hợp số liệu mưa vào mô hình được tiến hành thông qua quá trình tiền xử lý (pre-processing) để tạo thành file dfs2 làm đầu vào cho mô hình MIKE SHE (Hình 4c). Bản đồ sử dụng đất được xử lý từ bản đồ sử dụng đất toàn quốc (Atlas Việt nam) trong đó tiến hành thiết lập phân loại đất phù hợp đối với lưu vực nghiên cứu (Hình $4 \mathrm{~d})$. Bản đồ thảm phủ thực vật được xử lý từ bản đồ thảm phủ thực vật toàn quốc (Hình 4e).
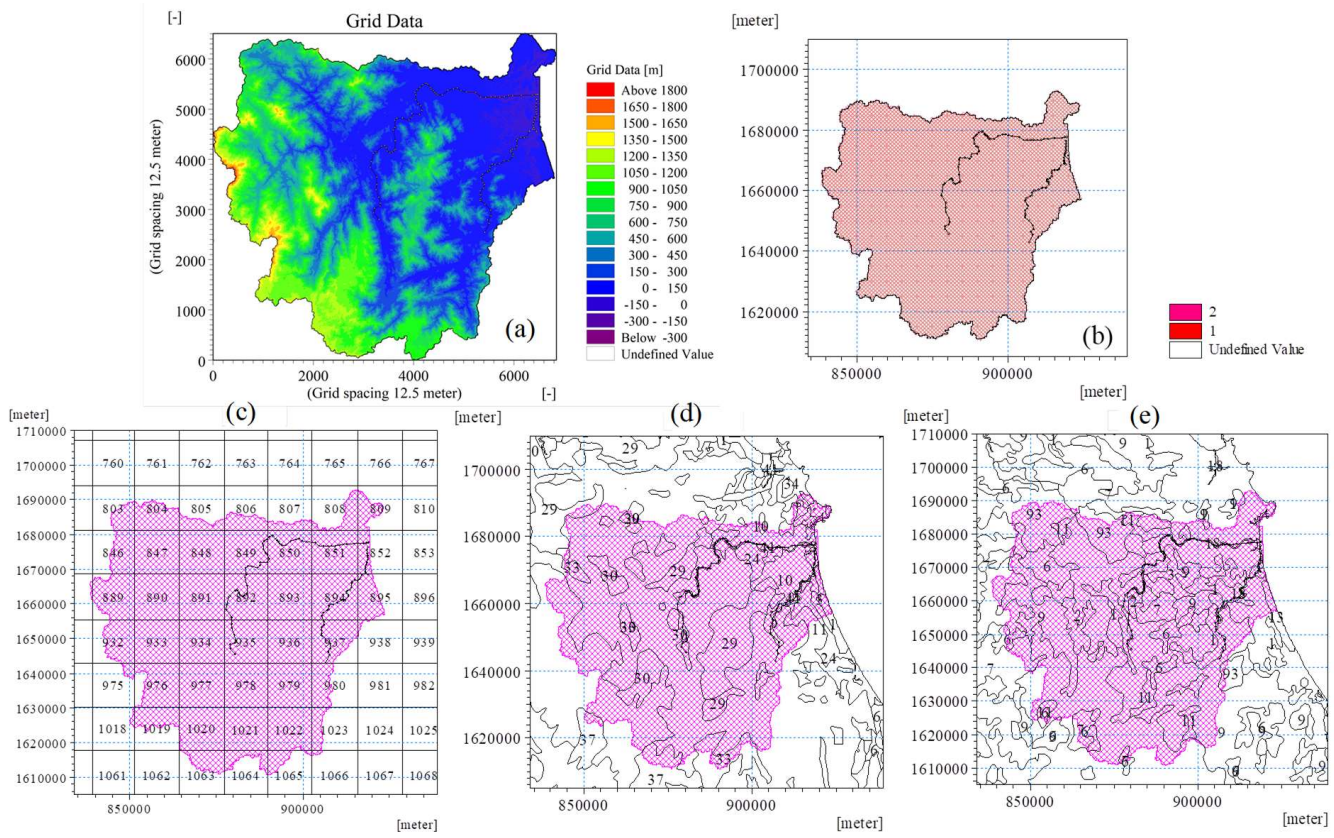

Hình 4. (a) Thiết lập địa hình vùng tính; (b) Thiết lập vùng tính và lưới tính; (c) Thiết lập biên khi tượng; (d)Thiết lập phân loại đất; (e)Thiết lập các thông số thảm phủ thực vật.

\section{Kết quả và thảo luận}

\subsection{Hiệu chỉnh và kiểm định mô hình}

Quá trình hiệu chỉnh mô hình được lựa chọn với 03 trận lũ điển hình tháng 10 năm 2012, tháng 10 năm 2013 và tháng 11 năm 2013 (Hình 5). Quá trình kiểm nghiệm mô hình được lựa chọn với 03 trận lũ điển hình tháng 11/2015, tháng 11/2016 và tháng 12/2016 (Hình 6). Hai trạm được lựa chọn để phục vụ hiệu chỉnh và kiểm định mô hình là trạm Sơn Giang trên lưu vực sông Trà Khúc và trạm An Chỉ trên lưu vực sông Vệ. Để đánh giá kết quả hiệu chỉnh và kiểm nghiệm mô hình nghiên cứu đã sử dụng 06 thông số để đánh giá: r, R2, NSE, RMSE, MAE, và PBIAS. Trong đó giá trị $r$ dao động từ 3,14 đến 6,25 trong cả hai quá trình hiệu chỉnh và kiểm định mô hình. Giá trị R2 dao động từ 0,81 đến 0,87 . Chỉ số Nash đạt giá trị dao động từ 0,80 đến 0,86 . Giá trị RMSE dao động từ 5,5 đến 23,9. Giá trị PBIAS dao động từ $-7,8 \%$ đến $9,84 \%$ trong cả hai quá trình hiệu chỉnh và kiểm định mô hình (Bảng 1). Kết quả tính toán chỉ ra 
rằng mức độ trung bình của các giá trị mô phỏng dòng chảy được đánh giá là rất tốt (PBIAS < $\pm 10)$ trong cả hai quá trình hiệu chỉnh và kiểm định mô hình. Bộ thông số của mô hình MIKE

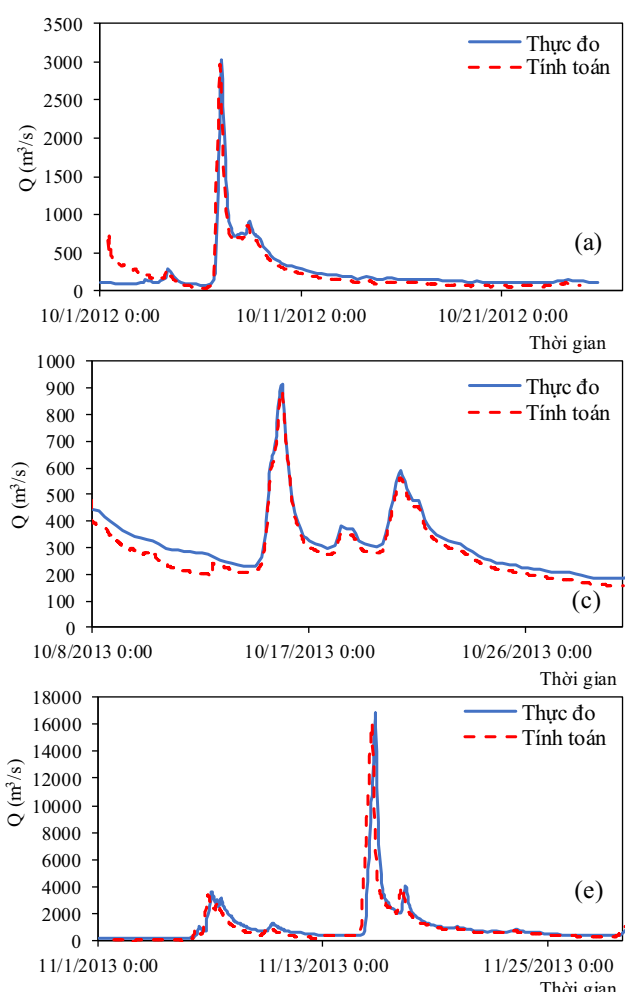

SHE trong quá trình hiệu chỉnh và kiểm định được sử dụng để tính toán, dự báo lưu lượng nước đến hai hồ ĐăkĐrinh và hồ Nước Trong trên lưu vực sông Trà Khúc-Sông Vệ (Bảng 2).

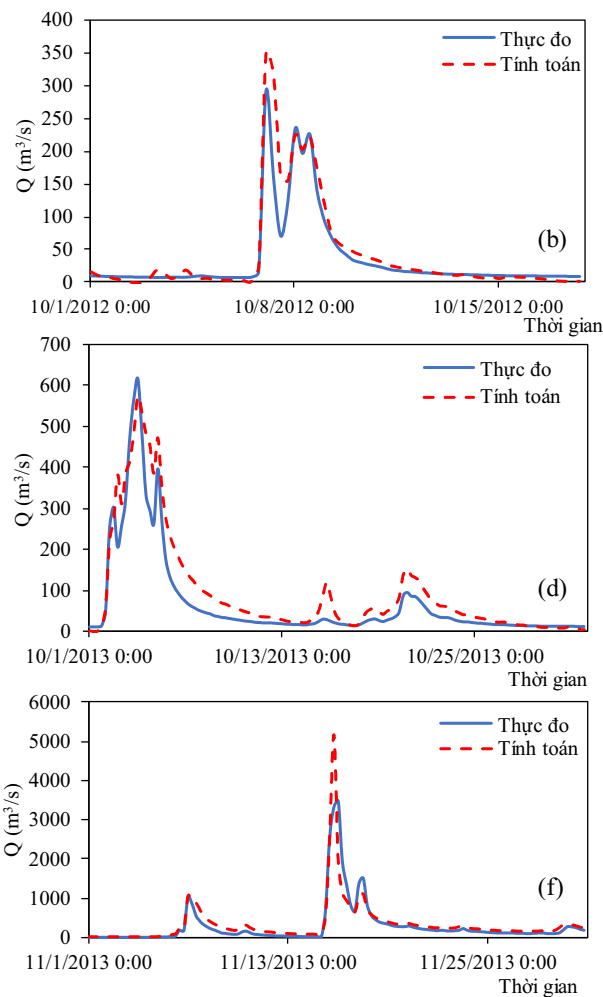

Hình 5. Kết quả hiệu chỉnh mô hình với 03 trận lũ năm 2012-2013 tại hai trạm: (a, c, e) Sơn Giang; (b, d, f) An Chi.
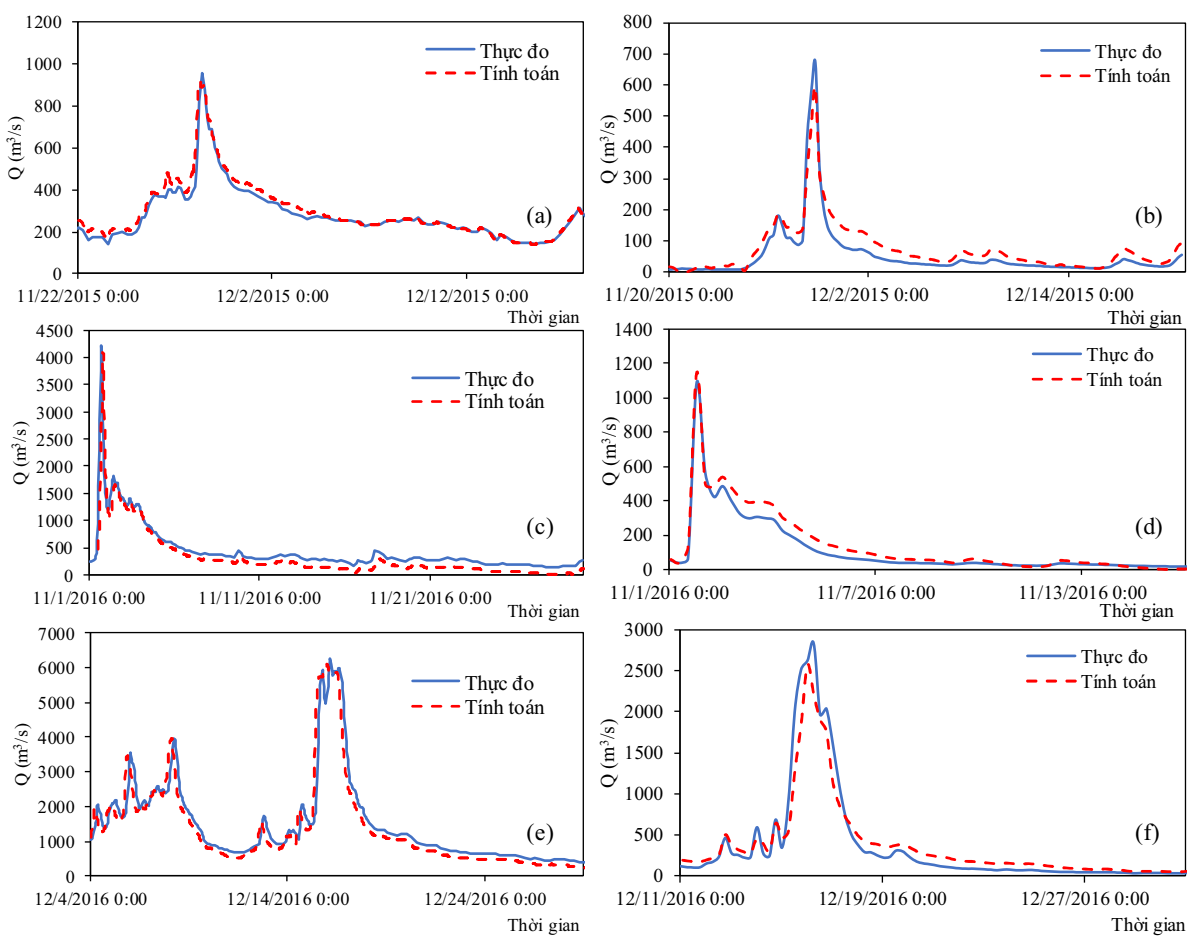

Hình 6. Kết quả kiểm định mô hình với 03 trận lũ năm 2015-2016 tại hai trạm: (a, c, e) Sơn Giang; (b, d, f)An Chi 


\section{BÀI BÁO KHOA HỌC}

Bảng 1. Tổng hợp kết quả đánh giá trong quá trình hiệu chỉnh và kiểm định mô hình

\begin{tabular}{|c|c|c|c|c|c|c|c|c|c|}
\hline $\begin{array}{l}\text { Quá } \\
\text { trình }\end{array}$ & Trận lũ & $\begin{array}{l}\text { Chỉ } \\
\text { tiêu }\end{array}$ & $\begin{array}{c}\text { Sơn } \\
\text { Giang }\end{array}$ & $\begin{array}{l}\text { An } \\
\text { Chỉ }\end{array}$ & $\begin{array}{l}\text { Quá } \\
\text { trình }\end{array}$ & Trận lũ & $\begin{array}{l}\text { Chỉ } \\
\text { tiêu }\end{array}$ & $\begin{array}{c}\text { Sơn } \\
\text { Giang }\end{array}$ & $\begin{array}{l}\text { An } \\
\text { Chỉ }\end{array}$ \\
\hline \multirow{18}{*}{$\begin{array}{l}\text { Hiệu } \\
\text { chỉnh }\end{array}$} & \multirow{6}{*}{$10 / 2012$} & $\mathrm{r}$ & 5,17 & 2,62 & \multirow{18}{*}{$\begin{array}{l}\text { Kiểm } \\
\text { định }\end{array}$} & \multirow{6}{*}{$11 / 2015$} & $\mathrm{r}$ & 4,7 & 2,19 \\
\hline & & $\mathrm{R}^{2}$ & 0,87 & 0,91 & & & $\mathrm{R}^{2}$ & 0,86 & 0,93 \\
\hline & & NSE & 0,86 & 0,81 & & & NSE & 0,84 & 0,87 \\
\hline & & RMSE & 23,9 & 19,7 & & & RMSE & 10,4 & 8,5 \\
\hline & & MAE & 18,2 & 9,1 & & & MAE & 19,6 & 5,6 \\
\hline & & PBIAS & 9,84 & $-6,5$ & & & PBIAS & $-4,96$ & $-3,7$ \\
\hline & \multirow{6}{*}{$10 / 2013$} & $\mathrm{r}$ & 6,25 & 3,5 & & \multirow{6}{*}{$11 / 2016$} & $\mathrm{r}$ & 4,15 & 2,5 \\
\hline & & $\mathrm{R}^{2}$ & 0,84 & 0,91 & & & $\mathrm{R}^{2}$ & 0,81 & 0,93 \\
\hline & & NSE & 0,83 & 0,84 & & & NSE & 0,8 & 0,89 \\
\hline & & RMSE & 6,67 & 5,2 & & & RMSE & 5,5 & 6,1 \\
\hline & & MAE & 16,5 & 12,39 & & & MAE & 15,4 & 7,9 \\
\hline & & PBIAS & $-0,18$ & $-3,8$ & & & PBIAS & 5,44 & $-4,6$ \\
\hline & \multirow{6}{*}{$11 / 2013$} & $\mathrm{r}$ & 5,47 & 2,07 & & \multirow{6}{*}{$12 / 2016$} & $\mathrm{r}$ & 3,14 & 4,01 \\
\hline & & $\mathrm{R}^{2}$ & 0,82 & 0,91 & & & $\mathrm{R}^{2}$ & 0,83 & 0,92 \\
\hline & & NSE & 0,84 & 0,8 & & & NSE & 0,86 & 0,86 \\
\hline & & RMSE & 8,63 & 8,9 & & & RMSE & 21,7 & 7,4 \\
\hline & & MAE & 10,25 & 9,4 & & & MAE & 17,1 & 8,9 \\
\hline & & PBIAS & 5,46 & $-7,8$ & & & PBIAS & 1,48 & 7,9 \\
\hline
\end{tabular}

Bảng 2. Thông số chính mô hình MIKE SHE lưu vục sông Trà Khúc - Sông Vệ

\begin{tabular}{|c|c|c|}
\hline Thông số chính & Đơn vị & Giá trị tối ưu \\
\hline \multicolumn{3}{|c|}{ Hệ số nhám lòng sông - Hệ số Strickler } \\
\hline Nhánh và thượng lưu Trà Khúc & $\mathrm{m}^{1 / 3} / \mathrm{s}$ & 18 \\
\hline Nhánh và thương lưu Sông Vệ & $\mathrm{m}^{1 / 3} / \mathrm{s}$ & 25 \\
\hline Nhánh kết nối & $\mathrm{m}^{1 / 3} / \mathrm{s}$ & 30 \\
\hline Hạ lưu sông & $\mathrm{m}^{1 / 3} / \mathrm{s}$ & 40 \\
\hline \multicolumn{3}{|l|}{ Dòng chảy tràn-Hệ số Strickler } \\
\hline Rừng trồng & $\mathrm{m}^{1 / 3} / \mathrm{s}$ & 5 \\
\hline Đất nông nghiệp & $\mathrm{m}^{1 / 3} / \mathrm{s}$ & 8 \\
\hline Lúa & $\mathrm{m}^{1 / 3} / \mathrm{s}$ & 16 \\
\hline Cây hàng năm & $\mathrm{m}^{1 / 3} / \mathrm{s}$ & 8 \\
\hline Cây lâu năm & $\mathrm{m}^{1 / 3} / \mathrm{s}$ & 8 \\
\hline Đất đã sử dụng & $\mathrm{m}^{1 / 3} / \mathrm{s}$ & 5 \\
\hline Rừng tự nhiên & $\mathrm{m}^{1 / 3} / \mathrm{s}$ & 2 \\
\hline Đô thị & $\mathrm{m}^{1 / 3} / \mathrm{s}$ & 90 \\
\hline Bề mặt nước & $\mathrm{m}^{1 / 3} / \mathrm{s}$ & 33 \\
\hline \multicolumn{3}{|c|}{ Dòng chảy không bão hoà - Độ rỗng của đất } \\
\hline $\mathrm{K}_{\mathrm{uz}-\mathrm{Sét}}$ & $\mathrm{m} / \mathrm{s}$ & $1.2 \times 10^{-8}$ \\
\hline $\mathrm{K}_{\mathrm{uz}}$-Sét bùn & $\mathrm{m} / \mathrm{s}$ & $2.45 \times 10^{-6}$ \\
\hline $\mathrm{K}_{\mathrm{uz}}$-cát mùn & $\mathrm{m} / \mathrm{s}$ & $8.5 \times 10^{-6}$ \\
\hline $\mathrm{K}_{\mathrm{uz}}$-Sét dẻo & $\mathrm{m} / \mathrm{s}$ & $2.085 \times 10^{-4}$ \\
\hline $\mathrm{K}_{\mathrm{uz}}$-Cát & $\mathrm{m} / \mathrm{s}$ & $2.89 \times 10^{-4}$ \\
\hline \multicolumn{3}{|l|}{ Vùng bão hoà } \\
\hline $\mathrm{K}_{\mathrm{h}}$ - Hệ số thấm theo chiều ngang & $\mathrm{m} / \mathrm{s}$ & $6.7 \times 10^{-5}$ \\
\hline
\end{tabular}

3.2 Đánh giá chất lượng kiểm định và dư kiểm định lại lưu lượng nước đến hai hồ báo lư lự̛ng đến hồ

ĐăkĐrinh và hồ Nước Trong trong hai trận lũ

Để phục vụ việc đánh giá chất lượng tính toán tháng 11/2016 và tháng 12/2016 (Hình 7). của mô hình, nhóm nghiên cứu đã tiến hành Nghiên cứu đã sử dụng 05 chỉ số để đánh giá $r$, 
$\mathrm{R}^{2}$, NSE, RMSE, MAE, PBIAS để so sánh và đánh giá kết quả tính toán và thực đo từ mô hình. Trong đó, giá trị $\mathrm{r}$ dao động từ $1,87-2,66 ; \mathrm{R}^{2}$ dao động từ $0,84-0,87$; NSE dao động từ $0,83-0,86$; RMSE dao động từ 4,2-11,6; MAE dao động từ 6,9-9,6; PBIAS dao động từ 1,1-5.5 đối với cả hai trận lũ năm 2016. Các kết quả đánh giá kiểm định đối với 02 trận lũ chỉ ra rằng có sự tương đồng cao về pha và biên độ dao động đối với đường quá trình tính toán và thực đo. Chất lượng
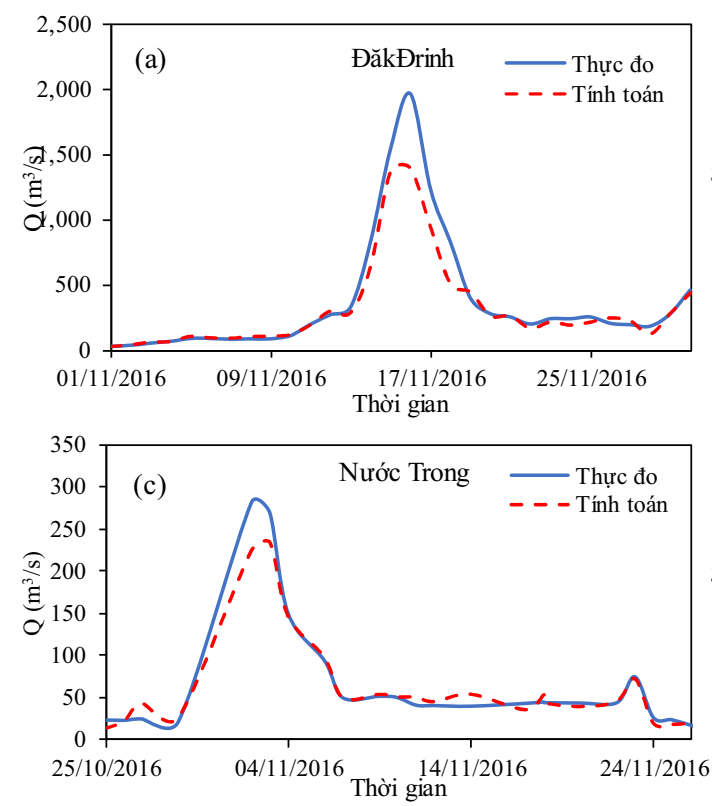

đánh giá mô hình cho cả hai trận lũ năm 2016 với các chỉ tiêu đánh giá $r, R^{2}$, NSE, RMSE, MAE, PBIAS cho kết quả rất tốt. Mô hình MIKE SHE tiếp tục được sử dụng để dự báo lưu lượng nước đến cho hai hồ ĐăkĐrinh và hồ Nước Trong trong hai trận lũ tháng 11/2017 và tháng 12/2017 (Hình 8). Kết quả đánh giá dự báo thử nghiệm cho kết quả tốt với chỉ tiêu NSE $>0,82$; $1,3<\mathrm{r}<7,6 ; \mathrm{R}^{2}>0,85 ;$ PBIAS $< \pm 10 \%$ (rất tốt) (Bảng 3).
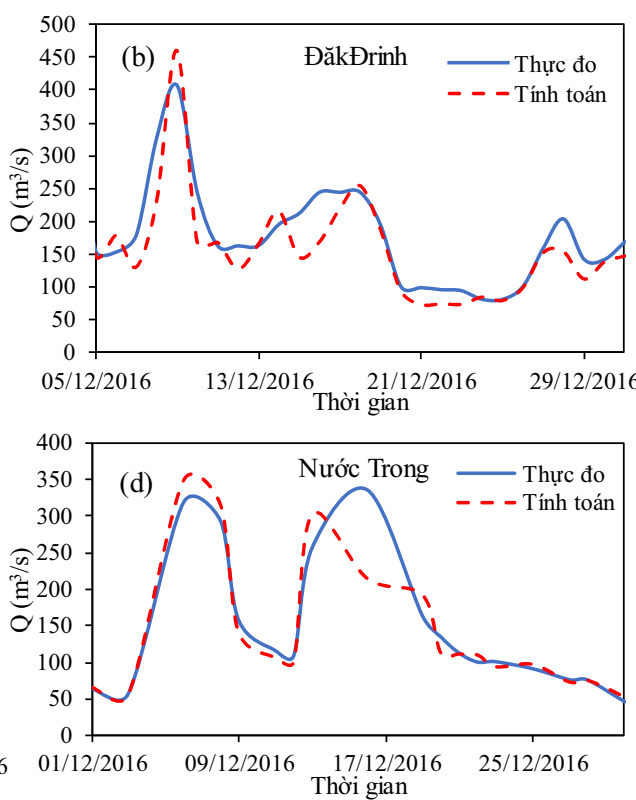

Hình 7. Kết quả kiểm định lưu lương nước đến hai hồ ĐăkĐrinh và hồ Nước Trong trong hai trận lũ tháng 11/2016 và tháng 12/2016

Bảng 3. Kết quả kiểm định và dụ báo luu lương nước đến hồ ĐăkĐrinh và hồ Nước Trong

\begin{tabular}{|c|c|c|c|c|c|c|c|c|c|}
\hline $\begin{array}{l}\text { Quá } \\
\text { trình }\end{array}$ & Năm & $\begin{array}{l}\text { Chỉ } \\
\text { tiêu }\end{array}$ & ĐăkĐrinh & $\begin{array}{l}\text { Nước } \\
\text { Trong }\end{array}$ & $\begin{array}{l}\text { Quá } \\
\text { trình }\end{array}$ & Năm & $\begin{array}{l}\text { Chỉ } \\
\text { tiêu }\end{array}$ & ĐăkĐrinh & $\begin{array}{l}\text { Nước } \\
\text { Trong }\end{array}$ \\
\hline \multirow{12}{*}{$\begin{array}{l}\text { Kiểm } \\
\text { định }\end{array}$} & \multirow{6}{*}{$11 / 2016$} & $r$ & 2,22 & 1,87 & \multirow{12}{*}{$\begin{array}{l}\text { Dự } \\
\text { báo }\end{array}$} & \multirow{6}{*}{$11 / 2017$} & $r$ & 3,7 & 3,4 \\
\hline & & $\mathrm{R}^{2}$ & 0,85 & 0,84 & & & $\mathrm{R}^{2}$ & 0,86 & 0,88 \\
\hline & & NSE & 0,83 & 0,84 & & & NSE & 0,82 & 0,84 \\
\hline & & RMSE & 4,2 & 10,9 & & & RMSE & 6,7 & 8,9 \\
\hline & & MAE & 6,9 & 9,6 & & & MAE & 9,4 & 13,7 \\
\hline & & PBIAS & 5,5 & 1,1 & & & PBIAS & 3,8 & 5,5 \\
\hline & \multirow{6}{*}{$12 / 2016$} & $\mathrm{r}$ & 2,27 & 2,66 & & \multirow{6}{*}{$12 / 2017$} & $\mathrm{r}$ & 1,3 & 7,6 \\
\hline & & $\mathrm{R}^{2}$ & 0,84 & 0,87 & & & $\mathrm{R}^{2}$ & 0,85 & 0,87 \\
\hline & & NSE & 0,85 & 0,86 & & & NSE & 0,83 & 0,85 \\
\hline & & RMSE & 8,5 & 11,6 & & & RMSE & 13,4 & 8,2 \\
\hline & & MAE & 7,9 & 8,9 & & & MAE & 2,46 & 9,7 \\
\hline & & PBIAS & 4,9 & 2,13 & & & PBIAS & 4,19 & 1,7 \\
\hline
\end{tabular}



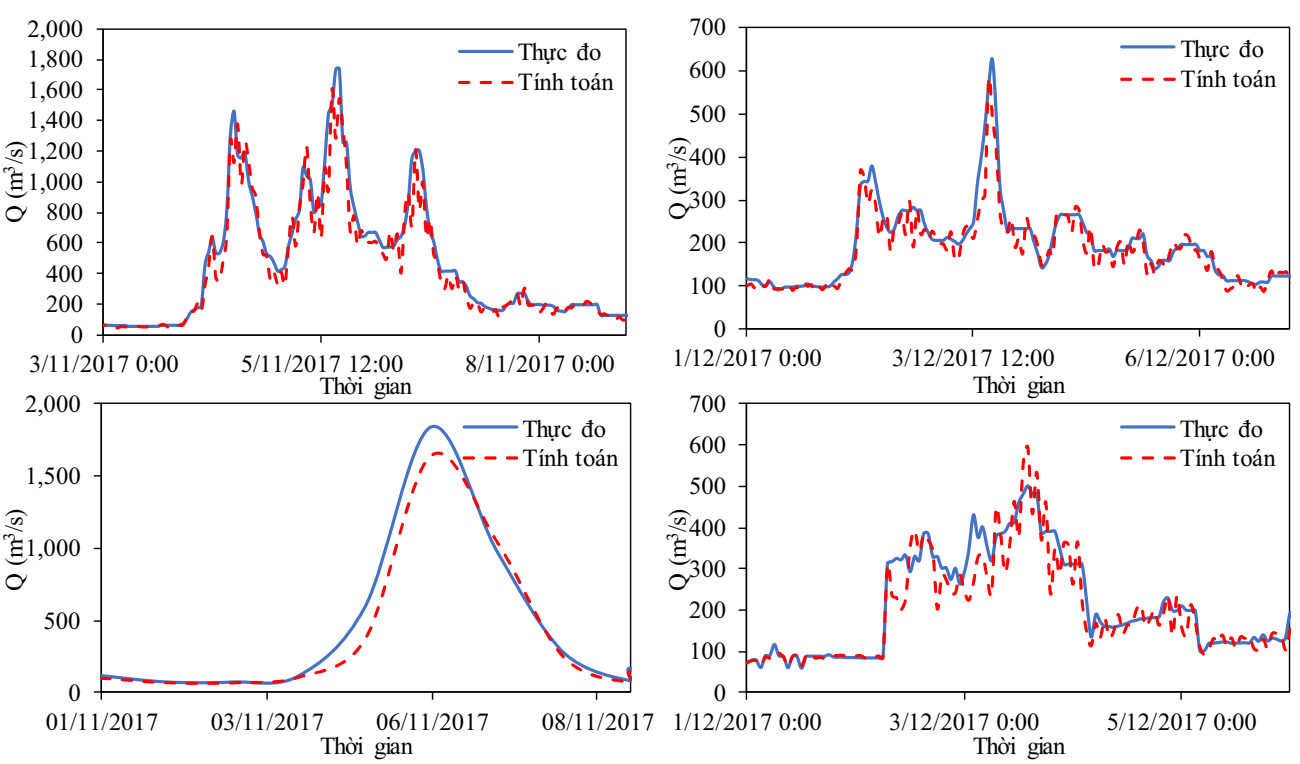

Hình 8. Kết quả thư nghiệm dụ báo lư lương nước đến hai hồ ĐăkĐrinh và hồ Nước Trong.

\section{Kết luận}

Nghiên cứu đã bước đầu thử nghiệm thành công việc kết hợp sản phẩm mưa dự báo từ mô hình IFS làm đầu vào cho mô hình thủy văn thông số phân bố MIKE SHE, phục vụ dự báo lưu lượng nước đến hai hồ ĐăkĐrinh và hồ Nước trong trên lưu vực sông Trà Khúc-Sông Vệ. Kết quả đánh giá bước đầu cho thấy chất lượng dự báo lưu lượng nước đến các hồ đã nâng lên đáng kể dựa trên các chỉ tiêu đánh giá chất lượng của mô hình r, R², NSE, RMSE, MAE, PBIAS. Đây có thể sẽ là một bộ công cụ hiệu quả được sử dụng tại phòng tác nghiệp của Trung tâm Dự báo
Khí tượng Thủy văn quốc gia và các sản phẩm dự báo sẽ hỗ trợ cho các đài KTTV khu vực cung cấp các bản tin phục vụ quy trình vận hành liên hồ chứa tới các hồ.

Lời cảm ơn: Bài báo là một phần kết quả của luận văn thạc sỹ của học viên Trần Đỗ Thủy Tuyên. Nghiên cứu này là một phần kết quả của đề tài nghiên cứu khoa học và phát triển công nghệ cấp Bộ: "Nghiên cứu ứng dụng số liệu dự báo của Trung tâm Dự báo thời tiết hạn vừa Châu Âu để xây dựng các phương án dự báo lũ 5 ngày cho các sông chính ở Trung Trung Bộ", mã số: TNMT.2018.05.35

\section{Tài liệu tham khảo}

1. Abbott, M.B., Bathurst, J.C., Cunge, J.A., O’Connell, P.E., Rasmussen, J. (1986a), An introduction to the European Hydrological System-Systeme Hydrologique Europeen, "SHE”, 1: history and philosophy of a physically-based, distributed modelling system. J. Hydrol. 87, 45-59.

2. Abbott, M.B., Bathurst, J.C., Cunge, J.A., O'connell, P.E., Rasmussen, J. (1986b), An introduction to the European Hydrological System Systeme Hydrologique Europeen, "SHE", 2: Structure of a physically-based, distributed modelling system. J. Hydrol. 87, 61-77.

3. Addiscott, T.M., Whitmore, A.P. (1987), Computer simulation of changes in soil mineral nitrogen and crop nitrogen during autumn, winter and spring. J. Agric. Sci. 109, 141-157.

4. Andersen, J., Refsgaard, J.C., Jensen, K.H. (2001), Distributed hydrological modelling of the Senegal River basin Model construction and validation. J. Hydrol. 247, 200-214.

5. Alansi, A.W., Amin, M.S.M., Abdul Halim, G., Shafri, H.Z.M., Aimrun, W. (2009), Validation of SWAT model for stream flow simulation and forecasting in Upper Bernam humid tropical river basin, Malaysia. Hydrol. Earth Syst. Sci. 6, 7581-7609. 
6. Adnan, N.A., Atkinson, P.M. (2018), Disentangling the effects of longterm changes in precipitation and land use on hydrological response in a monsoonal catchment. J. Flood Risk Manag. $11,1063-1077$.

7. Bathurst, J.C., Ewen, J., Parkin, G., O’Connell, P.E., Cooper, J.D. (2004), Validation of catchment models for predicting land-use and climate change impacts. 3. Blind validation for internal and outlet responses. J. Hydrol. 287, 74-94.

8. Dự án nhiệm vụ chuyên môn (2013-2015), Xây dựng công nghệ dụ báo lũ hạn ngắn phục vụ điều tiết hồ chứa cho lưu vực sông Vu Gia - Thu Bồn và lưu vực sông Ba.

9. Danish Hydraulic Institute (DHI), 2014a, MIKE SHE User Manual, Volume 1: User Guide, $370 \mathrm{p}$.

10. Danish Hydraulic Institute (DHI), 2014b, MIKE SHE User Manual, Volume 2: Reference Guide, $444 \mathrm{p}$.

11. Engel, B.A., Flanagan, D.C. (2006), Modeling and risk analysis of nonpoint-source pollution caused by atrazine using SWAT. Trans. ASABE 49, 667-678.

12. Gupta, H.V., Sorooshian, S., Yapo, P.O. (1999), Status of automatic calibration for hydrologic models: comparison with multilevel expert calibration. J. Hydrol. Eng. 4, 135-143.

13. Hoàng Anh Huy (2016), Nghiên cứu khả năng úng dụng mô hình Mike-She để mô phỏng độ ẩm trong đất, áp dụng thí điểm cho dòng chính khu vực sông La.

14. Jakeman, A.J., Letcher, R.A., Norton, J.P. (2006), Ten iterative steps in development and evaluation of environmental models. Environ. Modell. Softw. 21, 602-614.

15. Krause, P., Boyle, D.P., Bäse, F. (2005), Comparison of different efciency criteria for hydrological model assessment. Adv. Geosci. 5, 89-97.

16. Kwin, C.T., Talei, A., Alaghmand, S., Chua, L.H.C. (2016), Rainfall-runoff modeling using dynamic evolving neural fuzzy inference system with online learning. Proc. Eng. 154, 1103-1109.

17. Moriasi, D., Wilson, B. (2012), Hydrologic and water quality models: use, calibration, and validation. Trans. ASABE 55, 1241-1247.

18. Moriasi, D.N., Arnold, J.G., Liew, M.W. et al. (2007), Model evaluation guidelines for systematic quantifcation of accuracy in watershed simulations. Trans. ASABE 50, 885-900.

19. McCuen, R.H., Knight, Z., Cutter, A.G. (2006), Evaluation of the Nash-Sutcliffe efciency Index. J. Hydrol. Eng. 11, 597-602.

20. Mustafa, Y.M., Amin, M.S.M., Lee, T.S., Shariff, A.R.M. (2012), Evaluation of land development impact on a tropical watershed hydrology using remote sensing and GIS. J. Spat. Hydrol. 5, 16-30.

21. Ngô Đức Chân (2015), Nghiên cứu xác định lượng cung cấp thấm tì các hồ chứa nước lớn cho các tầng chứa nước ở Đông Nam Bộ bằng phưong pháp mô hình số. Áp dụng thư nghiệm cho hồ Dầu Tiếng.

22. Nejadhashemi, A.P., Wardynski, B.J., Munoz, J.D. (2011), Evaluating the impacts of land use changes on hydrologic responses in the agricultural regions of Michigan and Wisconsin. Hydrol. Earth Syst. Sci. Disc. 8, 3421-3468.

23. Nash, J.E., Sutcliffe, J.V. (1970), River flow forecasting through conceptual models part I-a discussion of principles. J. Hydrol. 10, 282-290.

24. Santhi, C., Arnold, J.G., Williams, J.R., Hauck, L.M., Dugas, W.A. (2001), Application of a watershed model to evaluate management effects on point and nonpoint source pollution. Trans. ASABE 44, 1559-1570.

25. Vũ Đức Long (2014), Nghiên cứu xây dụng công nghệ cảnh báo, dụ báo lũ và cảnh báo ngập 


\title{
BÀI BÁO KHOA HỌC
}

lụt cho các sông chính ở Quảng Bình, Quảng Trị.

26. Vũ Đức Long (2015), Nghiên cưu ứng dụng thử nghiệm mô hình TELEMAC 2D tính toán lũ và cảnh báo ngập lụt cho vùng hạ lưu sông Trà Khúc - Sông Vệ.

27. Vertex. https://vertex.daac.asf.alaska.edu/

28. Wong, C.L., Venneker, R., Uhlenbrook, S. (2010), Analysis and modelling of runoff from two distinct river basins in Peninsular Malaysia. In: HydroPredict conference, p. 11.

29. Wang, L., Koike, T., Yang, K., Jackson, T.J., Bindlish, R., Yang, D. (2008), WEB-DHM: A distributed biosphere hydrological model developed by coupling a simple biosphere scheme with a hillslope hydrological model. American Geophysical Union, Fall Meeting 2008, abstract id. H32D-08.

\section{APPLICATION MIKE SHE MODEL COMBINED WITH RAINFALL FORECASTING PRODUCT (IFS) TO FORECAST INFLOW TO RESERVOIRS ON TRA KHUC - SONG VE BASIN}

\author{
Tran Hong Thai ${ }^{1}$, Doan Quang Tri' ${ }^{2}$, Tran Do Thuy Tuyen ${ }^{3}$, \\ Ngo Thanh Tam², Bui Thi Diu \\ ${ }^{1}$ Viet Nam Meteorological and Hydrometeorological Administration, Ha Noi, Vietnam \\ ${ }^{2}$ Vietnam Journal of Hydrometeorology, Viet Nam Meteorological and \\ Hydrometeorological Administration, Ha Noi, Vietnam \\ ${ }^{3}$ Hanoi University of Natural Resources and Environment, Ha Noi, Vietnam
}

\begin{abstract}
The paper has applied the IFS products to calculate and forecast the inflow to two reservoirs on Tra Khuc - Song Ve basin. The process of calibration and validation hydrological models is regulated by the input data of typical floods on the basin from the period of 2013 - 2016. The results of these processes indicate the similarity between calculated and measured discharge at two stations including Son Giang and An Chi. These results then be used to cablirate and forecast the inflow to two reservoirs including DakDrinh and Nuoc Trong. The outcomes are relatively qualified in accordance with several criterials such as r, R2, NSE, RMSE, MAE and PBIAS. Experimental results for the two typical floods in 2017 indicate that the quality of forecasted flows to the two reservoirs has increased significantly. This is supposed to be an effective tool for forecastors in the future.
\end{abstract}

Keywords: IFS, MIKE SHE, Tra Khuc-Song Ve. 